\title{
Modulational instability in nonlocal nonlinear Kerr media
}

\author{
Krolikowski, Wieslaw; Bang, Ole; Juul Rasmussen, Jens; Wyller, John
}

Published in:

Physical Review E. Statistical, Nonlinear, and Soft Matter Physics

Link to article, DOI:

10.1103/PhysRevE.64.016612

Publication date:

2001

Document Version

Publisher's PDF, also known as Version of record

Link back to DTU Orbit

Citation (APA):

Krolikowski, W., Bang, O., Juul Rasmussen, J., \& Wyller, J. (2001). Modulational instability in nonlocal nonlinear Kerr media. Physical Review E. Statistical, Nonlinear, and Soft Matter Physics, 64(1), 016612.

https://doi.org/10.1103/PhysRevE.64.016612

\section{General rights}

Copyright and moral rights for the publications made accessible in the public portal are retained by the authors and/or other copyright owners and it is a condition of accessing publications that users recognise and abide by the legal requirements associated with these rights.

- Users may download and print one copy of any publication from the public portal for the purpose of private study or research.

- You may not further distribute the material or use it for any profit-making activity or commercial gain

- You may freely distribute the URL identifying the publication in the public portal

If you believe that this document breaches copyright please contact us providing details, and we will remove access to the work immediately and investigate your claim. 


\title{
Modulational instability in nonlocal nonlinear Kerr media
}

\author{
Wieslaw Krolikowski \\ Australian Photonics Cooperative Research Centre, Laser Physics Centre, Research School of Physical Sciences and Engineering, \\ Australian National University, Canberra ACT 0200, Australia \\ Ole Bang \\ Department of Informatics and Mathematical Modeling, Technical University of Denmark, DK-2800 Kongens Lyngby, Denmark \\ Jens Juul Rasmussen \\ Risb National Laboratory, Optics and Fluid Dynamics Department, OFD - 128, P.O. Box 49, DK-4000 Roskilde, Denmark \\ John Wyller \\ Department of Mathematical Sciences, Agricultural University of Norway, P.O. Box 5065, N-1432 Ås, Norway
}

(Received 4 March 2001; published 26 June 2001)

\begin{abstract}
We study modulational instability (MI) of plane waves in nonlocal nonlinear Kerr media. For a focusing nonlinearity we show that, although the nonlocality tends to suppress MI, it can never remove it completely, irrespective of the particular profile of the nonlocal response function. For a defocusing nonlinearity the stability properties depend sensitively on the response function profile: for a smooth profile (e.g., a Gaussian) plane waves are always stable, but MI may occur for a rectangular response. We also find that the reduced model for a weak nonlocality predicts MI in defocusing media for arbitrary response profiles, as long as the intensity exceeds a certain critical value. However, it appears that this regime of MI is beyond the validity of the reduced model, if it is to represent the weakly nonlocal limit of a general nonlocal nonlinearity, as in optics and the theory of Bose-Einstein condensates.
\end{abstract}

DOI: 10.1103/PhysRevE.64.016612

PACS number(s): 42.65.Tg, 52.35.Mw, 42.65.Jx, 42.25.Bs

\section{INTRODUCTION}

Modulational instability (MI) constitutes one of the most fundamental effects associated with wave propagation in nonlinear media. It signifies the exponential growth of a weak perturbation of the amplitude of the wave as it propagates. The gain leads to amplification of sidebands, which breaks up the otherwise uniform wave front and generates fine localized structures (filamentation). Thus it may act as a precursor for the formation of bright spatial solitons. Conversely the generation of dark spatial solitons requires the absence of MI of the constant intensity background.

The phenomena of MI has been identified and studied in various physical systems, such as fluids [1], plasma [2], nonlinear optics $[3,4]$, discrete nonlinear systems, such as molecular chains [5] and Fermi-resonant interfaces and waveguide arrays [6], etc. It has been shown that MI is strongly affected by various mechanisms present in nonlinear systems, such as higher order dispersive terms in case of optical pulses [7], saturation of the nonlinearity [8], and coherence properties of optical beams [9].

In this work we study the MI of plane waves propagating in a nonlinear Kerr-type medium with a nonlinearity (the refractive index change $\Delta n$, in nonlinear optics) that is a nonlocal function of the incident field. We consider a phenomenological model, in which the nonlocal nonlinearity, induced by a wave (e.g., an optical beam) with the intensity $I(x, z)$, can be represented in general form as

$$
\Delta n(I)=s \int_{-\infty}^{\infty} R\left(x^{\prime}-x\right) I\left(x^{\prime}, z\right) d x^{\prime}
$$

where $x$ is the transverse spatial coordinate and $s=1 \quad(s=$ $-1)$ corresponds to a focusing (defocusing) nonlinearity. The evolution coordinate $z$ can be time, as for Bose-Einstein Condensates (BEC's), or the spatial propagation coordinate, as for optical beams. We consider only symmetric spatial response functions that are positive definite and (without loss of generality) obey the normalization condition

$$
\int_{-\infty}^{\infty} R(x) d x=1 .
$$

Thus we exclude asymmetric effects, such as those generated by asymmetric temporal response functions (with $x$ being time), as in the case of the Raman effect on optical pulses [10].

In nonlinear optics Eq. (1) represents a general phenomenological model for media in which the nonlinear refractive index change (or polarization) induced by an optical beam is determined by some kind of a transport process. It may include, e.g., heat conduction in materials with a thermal nonlinearity [11-13] or diffusion of molecules or atoms accompanying nonlinear light propagation in atomic vapors [14]. Nonlocality also accompanies the propagation of waves in plasma [15-19], and a nonlocal response in the form (1) appears naturally as a result of many body interaction processes in the description of Bose-Einstein condensates [20].

The width of the response function $R(x)$ relative to the width of the intensity profile $I(x, z)$ determines the degree of nonlocality, as illustrated in Fig. 1. In the limit of a singular response, $R(x)=\delta(x)$ [see Fig. 1(a)], the nonlinearity is a local function of the intensity, 


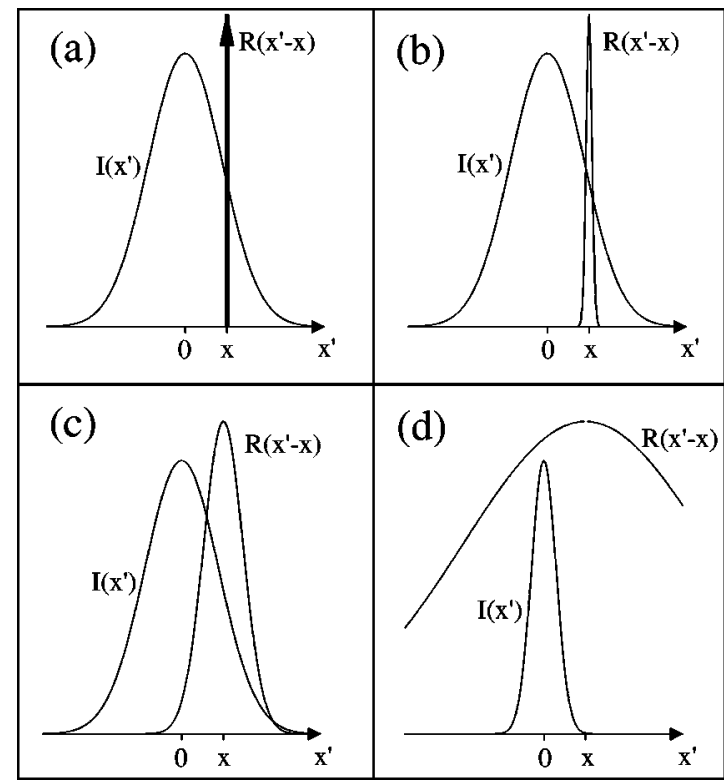

FIG. 1. Different degrees of nonlocality, as given by the width of the response function $R(x)$ and the intensity profile $I(x)$. Shown is the local (a), weakly nonlocal (b), general (c), and strongly nonlocal (d) response.

$$
\Delta n=s I,
$$

i.e., the refractive index change at a given point is solely determined by the wave amplitude at that very point. For this well-known Kerr nonlinearity, which appears in all areas of physics, MI depends only on the sign $s$ [3].

With increasing width of $R(x)$ the intensity in the vicinity of the point $x$ also contributes to the index change at that point. For a weak nonlocality, when the width of $R(x)$ is finite but still small compared to the width of $I(x, z)$ [see Fig. $1(\mathrm{~b})]$, one can expand $I\left(x^{\prime}, z\right)$ around $x^{\prime}=x$ in Eq. (1) and obtain the simplified model

$$
\Delta n=s\left(I+\gamma \partial_{x}^{2} I\right), \quad \gamma=\frac{1}{2} \int_{-\infty}^{\infty} x^{2} R(x) d x,
$$

where $\gamma$ is a small positive parameter. This diffusion type model of the nonlocal nonlinearity is a model in its own right in plasma physics, where $\gamma$ can take any sign [15-17]. It was also applied to BEC's [21], nonlinear optics [22] and in the continuum limit of the theory of energy transfer in biomolecular systems $[23,24]$. In contrast to the local Kerr limit the MI now depends not only on the sign $s$, but also on the intensity of the plane wave [16].

In the limit of a strongly nonlocal response, where $R(x)$ is much broader than the intensity profile [see Fig. 1(d)], one can expand the response function around $x^{\prime}=x$ in Eq. (1) and obtain the simple linear model

$$
\Delta n=s\left(c_{0}+c_{1} x+c_{2} x^{2}\right),
$$

where $c_{0-2}$ are constants. Since this model is linear all plane waves are stable-there is no MI. The evolution of optical beams in such a medium was considered in the case when $c_{1}=0$ [25], which requires the center of mass of the beam to be always zero.

Modulational instability has thus been studied in the different limits. However, in the general case (1) the problem of MI has only been studied for a few particular cases of the response function $R(x)$. Here we present an analytical study of the general case with arbitrary profile $R(x)$ and confirm the results for three specific examples. We further show that the MI results of the weakly nonlocal model (4) lead to erroneous conclusions when used to predict the behavior of the full model (1).

\section{GENERAL THEORY}

We will consider an optical beam propagating along the $z$ axis, with the scalar amplitude of the electric field expressed as

$$
E(x, z)=\psi(x, z) \exp (i K z-i \Omega t)+\text { c.c. }
$$

where $K$ is the wave number, $\Omega$ is the optical frequency, and $\psi(x, z)$ is the slowly varying amplitude. Substituting Eqs. (1) and (6) into Maxwell's equations we obtain the nonlocal nonlinear Schrödinger (NLS) equation

$$
i \partial_{z} \psi+\frac{1}{2} \partial_{x}^{2} \psi+s \psi \int_{-\infty}^{\infty} R\left(x^{\prime}-x\right) I\left(x^{\prime}, z\right) d x^{\prime}=0
$$

where $I(x, z)=|\psi(x, z)|^{2}$ is the intensity of the beam. The model (7) permits plane wave solutions of the form

$$
\psi(x, z)=\sqrt{\rho_{0}} \exp \left(i k_{0} x-i \omega_{0} z\right), \quad \rho_{0}>0
$$

where $\rho_{0}, k_{0}$, and $\omega_{0}$ are linked through the nonlinear dispersion relation

$$
\omega_{0}=\frac{1}{2} k_{0}^{2}-s \rho_{0},
$$

which is the same as for the standard local NLS equation

$$
i \partial_{z} \psi+\frac{1}{2} \partial_{x}^{2} \psi+s|\psi|^{2} \psi=0
$$

Next, let us carry out a linear stability analysis of the plane wave solutions (8). Assume that

$$
\psi(x, z)=\left[\sqrt{\rho_{0}}+a_{1}(x, z)\right] \exp \left(i k_{0} x-i \omega_{0} z\right)
$$

where $a_{1}(x, z)$ is a small complex perturbation. Inserting this expression into the nonlocal NLS equation (7) and linearizing around the solution (8) yields the evolution equation for the perturbation

$$
i \partial_{\tau} a_{1}+\frac{1}{2} \partial_{\xi}^{2} a_{1}+2 s \rho_{0} \int_{-\infty}^{\infty} R\left(\xi^{\prime}-\xi\right) \operatorname{Re}\left\{a_{1}\left(\xi^{\prime}, \tau\right)\right\} d x^{\prime}=0 .
$$


In deriving this equation we have used the nonlinear dispersion relation (9) and gone into a coordinate frame moving with the group velocity $c_{g}=d \omega_{0} / d k_{0}=k_{0}$, i.e.,

$$
\tau=z, \quad \xi=x-c_{g} z .
$$

Decomposing the perturbation into real and imaginary parts, $a_{1}=u+i v$, we obtain two coupled equations

$$
\begin{gathered}
\partial_{\tau} u+\frac{1}{2} \partial_{\xi}^{2} v=0, \\
\partial_{\tau} v-\frac{1}{2} \partial_{\xi}^{2} u-2 s \rho_{0} \int_{-\infty}^{\infty} R\left(\xi^{\prime}-\xi\right) u\left(\xi^{\prime}, \tau\right) d \xi^{\prime}=0 .
\end{gathered}
$$

By introducing the Fourier transforms

$$
\begin{gathered}
\hat{u}(k, \tau)=\int_{-\infty}^{\infty} u(\xi, \tau) \exp [i k \xi] d \xi, \\
\hat{v}(k, \tau)=\int_{-\infty}^{\infty} v(\xi, \tau) \exp [i k \xi] d \xi, \\
\hat{R}(k)=\int_{-\infty}^{\infty} R(\xi) \exp [i k \xi] d \xi,
\end{gathered}
$$

and exploiting the convolution theorem for Fourier transforms, the linearized system is converted to a set of ordinary differential equations in $k$ space

$$
\begin{gathered}
\partial_{\tau} \hat{u}-\frac{1}{2} k^{2} \hat{v}=0, \\
\partial_{\tau} \hat{v}+\frac{1}{2} k^{2} \hat{u}-2 s \rho_{0} \hat{R} \hat{u}=0,
\end{gathered}
$$

which can be written in the compact matrix form

$$
\partial_{\tau} \underline{X}=\underline{A} \underline{X}
$$

where the vector $\underline{X}$ and matrix $\stackrel{A}{=}$ are defined as

$$
\underline{X}=\left[\begin{array}{l}
\hat{u} \\
\hat{v}
\end{array}\right], \quad \stackrel{A=}{=}\left[\begin{array}{cc}
0 & \frac{1}{2} k^{2} \\
-\frac{1}{2} k^{2}+2 s \rho_{0} \hat{R}(k) & 0
\end{array}\right] .
$$

The eigenvalues $\lambda$ of the matrix $\stackrel{A}{=}$ are given by

$$
\lambda^{2}=-k^{2} \rho_{0}\left[\alpha k^{2}-s \hat{R}(k)\right],
$$

where we have defined the parameter $\alpha$ as

$$
\alpha=1 /\left(4 \rho_{0}\right) .
$$

Notice that if the response function $R(x)$ is real and symmetric then so is the Fourier spectrum of $R(x)$, i.e., $\hat{R}(k)$ $=\hat{R}^{*}(k)=\hat{R}(-k)$.

The general eigenvalue equation (19) constitutes the basis of our further study. First of all we notice that $\hat{R}(0)=1$, since the response function is normalized according to Eq.
(2). For a focusing nonlinearity $(s=+1)$ we therefore always have, by continuity, that $\alpha k^{2}-s \hat{R}(k)<0$, and thus $\lambda^{2}>0$, in a certain wave number band symmetrically centered about the origin, where $k$ is sufficiently small. It follows that we always will have (long wave) MI in the focusing case, independently of the details in the behavior of the response function.

In contrast, in the defocusing case $(s=-1)$, the stability properties depend in a sensitive way on the response function. Thus the nonlocality could possibly lead to MI, which would never be present in the defocusing local NLS equation (10).

The well-known modulational instability (stability) result for the standard local NLS equation (10) is easily recovered from the general eigenvalue equation (19) by setting $R(x)$ $=\delta(x)$, where $\delta(x)$ is the Dirac $\delta$ function: We get

$$
\lambda^{2}=-k^{2} \rho_{0}\left(\alpha k^{2}-s\right)
$$

where $s=+1 \quad(s=-1)$ yields instability (stability).

\section{WEAKLY NONLOCAL LIMIT}

The MI properties in the local limit (3), described by the NLS equation (10), are well known and the strongly nonlocal limit (5) is linear, so obviously it does not display MI. Here we briefly consider the interesting regime of a weak nonlocality, i.e., the situation when the typical width of the response kernel is small compared to the characteristic length of the wave modulation (see Fig. 1). In this case the nonlinearity has the form (4) and the NLS equation (7) reads $[21,24]$

$$
i \partial_{z} \psi+\frac{1}{2} \partial_{x}^{2} \psi+s\left(|\psi|^{2}+\gamma \partial_{x}^{2}|\psi|^{2}\right) \psi=0,
$$

where the nonlocality or diffusion parameter $\gamma$ is defined in Eq. (4). Equation (22) has been discussed in the literature in the context of plasma physics [17] and the continuum limit of discrete molecular structures $[23,24]$ and has been shown to posses bright and dark soliton solutions. Their exact analytical soliton form was found recently [24]. Importantly, $\gamma$ is small and positive when Eq. (22) represents the weakly nonlocal limit of the general nonlocal model (7), whereas $\gamma$ can take any sign in plasma physics, where Eq. (22) is a model in its own right.

The instability condition for the weak nonlocality is most easily obtained by Taylor expanding the spectrum of the response kernel about $k=0$ and only retaining the two lowest order nontrivial terms in the expansion, i.e.,

$$
\hat{R}(k) \simeq 1-\gamma k^{2},
$$

where obviously $\gamma k^{2} \ll 1$ must be fulfilled. Physically this expansion means that the width of the response function is much narrower than the spatial scale of the intensity distribution. The eigenvalue equation then reads

$$
\lambda^{2}=-k^{2} \rho_{0}\left[(\alpha+s \gamma) k^{2}-s\right] .
$$


TABLE I. Modulational stability properties of the weakly nonlocal model (22).

\begin{tabular}{lcc}
\hline \hline & $s=1$ & $s=-1$ \\
\hline$\gamma<0$ & MI & Stability \\
\hline$\gamma>0$ & MI & Stability for $\rho_{0}<\rho_{0}^{\text {cr }}$ \\
MI for $\rho_{0}>\rho_{0}^{\text {cr }}$
\end{tabular}

This result does not depend on the detailed behavior of the response kernel, as should be expected.

The eigenvalue Eq. (24) clearly shows that MI is always present for a focusing nonlinearity $(s=+1)$, since for sufficiently small wave numbers $\lambda^{2}>0$ for all values of $\alpha$ and $\gamma$. This is in correspondence with the results of the general nonlocal model (7).

More interesting is the defocusing case $(s=-1)$, where there is a critical value $\rho_{0}^{\mathrm{cr}}$ of the intensity,

$$
\rho_{0}^{\mathrm{cr}}=1 /(4 \gamma)
$$

which depends on the degree of nonlocality through the parameter $\gamma$, and corresponds to $\alpha=\gamma$. When $\gamma$ is positive (and $s=-1$ ) low intensity plane waves with $\rho_{0} \leqslant \rho_{0}^{\text {cr }}$ are modulationally stable, whereas MI appears at high intensities, $\rho_{0}>\rho_{0}^{\mathrm{cr}}$, despite the nonlinearity being defocusing. This MI is short-wave, appearing for perturbations with wave numbers exceeding the threshold value

$$
k^{\mathrm{cr}}=1 / \sqrt{|\alpha-\gamma|} .
$$

The stability criterion $\rho_{0}<\rho_{0}^{\text {cr }}$ corresponds exactly to the criterion for existence of dark soliton solutions in this case [24]. The existence of MI in the weakly nonlocal model is in sharp contrast to the local defocusing Kerr nonlinearity, for which there is no MI.

In the case $\gamma<0$ in Eq. (22), relevant in plasma physics, nonlocality does not affect the MI properties: for a focusing (defocusing) nonlinearity all plane waves are modulationally unstable (stable). In Table I we have summarized the MI results of the weak nonlocality model.

So only for defocusing media with $\gamma>0$ does a weak nonlocality change the MI properties in terms of existence, as compared to the local NLS limit. However, nonlocality does always affect the structure of the MI gain spectrum, as illustrated in Fig. 2 for $\gamma>0$. In the focusing case Fig. 2 clearly shows how the nonlocality tends to suppress MI by decreasing both the maximum gain and the gain bandwidth. In contrast, in the defocusing case, the nonlocality promotes $M I$ - the higher the degree of nonlocality $\gamma$ the lower the threshold value of the wave number, above which the instability develops.

In Fig. 3 we show the development of MI of a plane wave in a self-defocusing weakly nonlocal medium, as obtained by numerical integration of the NLS equation (22). The numerical procedure involved the split-step Fourier method for field propagation combined with the finite difference for calculating the nonlocal term in Eq. (10). As an initial condition we

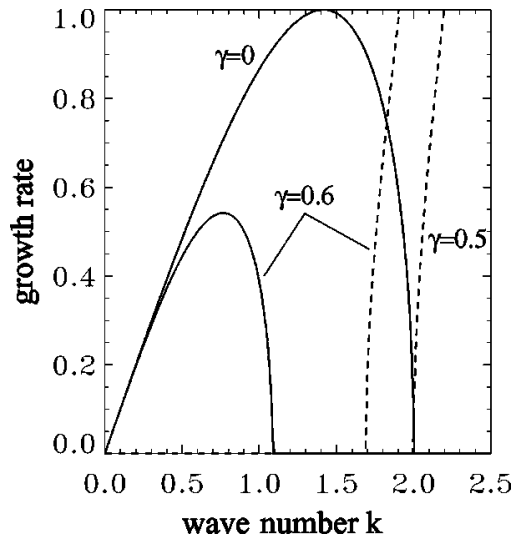

FIG. 2. Growth rate vs wave number for plane waves with intensity $\rho_{0}=1$ in media with a weakly nonlocal focusing $(s=1$, solid lines) and defocusing ( $s=-1$, dashed lines) nonlinearity.

used a plane wave with periodically perturbed amplitude. It is evident that this perturbation grows as the wave propagates.

Although Eqs. (25) and (26) predict MI in the defocusing case it is important to stress that this result does not actually apply to the case of weak nonlocality. In deriving the model (22) and the expansion (23) it was assumed that

$$
\gamma k^{2} \ll 1
$$

which means that the width of the response function must be small compared to the characteristic length scale of the modulation. However, from Eq. (24) we get that MI occurs when

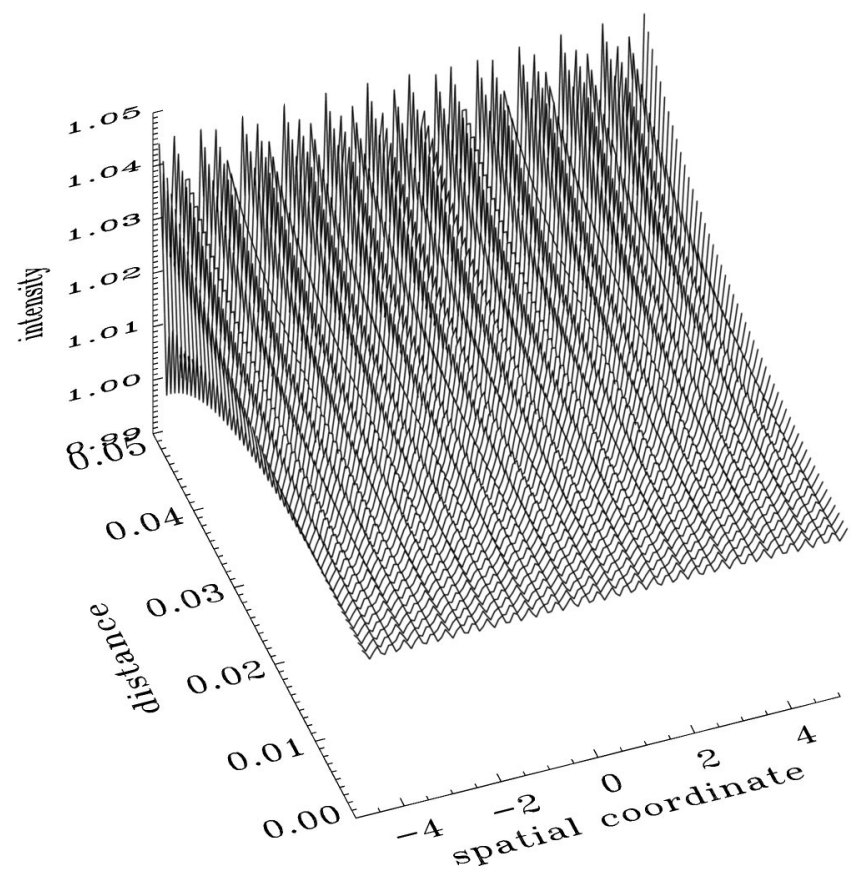

FIG. 3. Development of MI of a plane wave with intensity $\rho_{0}$ $=1$ in a weakly nonlocal defocusing $(\mathrm{s}=-1)$ medium. The initial amplitude is modulated with the wave vector $k=7 \pi$. 


$$
(\alpha-\gamma) k^{2}+1<0
$$

which can be rewritten as

$$
\gamma k^{2}>1+\alpha k^{2}
$$

Since $\alpha=1 /\left(4 \rho_{0}\right)$ is positive this condition cannot be fulfilled without violating the perturbation requirement (27). Hence, in the region of parameters where MI occurs (and dark solitons do not exist), the weak nonlocality approximation breaks down. This is in accordance with the analysis of the full nonlocal model (7), which, as we show below, does not display MI for self-defocusing nonlinearities with a smooth nonlocal response function.

It should be stressed however that the appearance of MI is a valid result in the situation when the weakly nonlocal model (7) is a model in its own right, as, for example, for upper hybrid modes in plasma [17].

\section{FULLY NONLOCAL RESPONSE-EXAMPLES}

We will now discuss the stability issue in the general nonlocal Kerr nonlinearity with an arbitrary degree of nonlocality by considering a few specific examples of the nonlocal response function.

\section{A. Gaussian response function}

Let the response function of the nonlocal nonlinear medium have the following Gaussian form

$$
R(x)=\frac{1}{\sigma \sqrt{\pi}} \exp \left[-\frac{x^{2}}{\sigma^{2}}\right] .
$$

In this case $\gamma=\sigma^{2} / 2$, the Fourier transform of the response function is given by

$$
\hat{R}(k)=\exp \left[-\frac{1}{4} \sigma^{2} k^{2}\right]
$$

and the eigenvalue equation (19) reduces to

$$
\lambda^{2}=-k^{2} \rho_{0}\left(\alpha k^{2}-s \exp \left[-\frac{1}{4} \sigma^{2} k^{2}\right]\right) .
$$

For the defocusing case $(s=-1)$ we find modulational stability for all values of the intensity $\rho_{0}$, in contrast to the behavior predicted by the weakly nonlocal model (22), according to which plane waves become unstable if their intensity is higher than the critical intensity $\rho_{0}^{\mathrm{cr}}$. Thus, as expected, this proves that the weakly nonlocal model gives incorrect predictions of the MI properties of the full general nonlocal model.

A simple analysis reveals finite bandwidth $\mathrm{MI}$ in the focusing case $(s=+1)$ with a growth rate given by

$$
|\operatorname{Re} \lambda|=|k| \sqrt{\rho_{0}} \sqrt{\exp \left[-\frac{1}{4} \sigma^{2} k^{2}\right]-\alpha k^{2}} .
$$

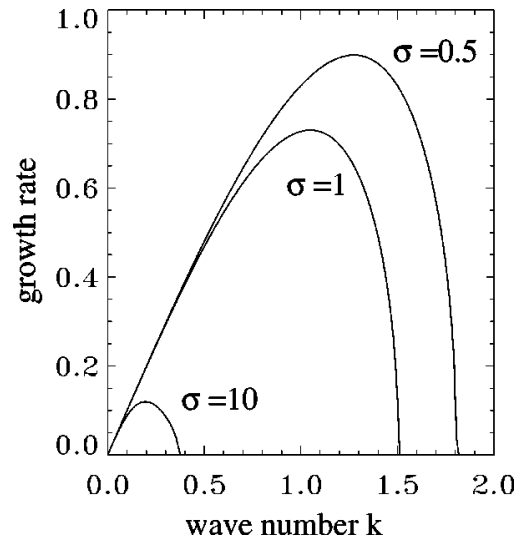

FIG. 4. MI gain profiles for $\rho_{0}=1$ in self-focusing ( $\left.\mathrm{s}=1\right)$ nonlocal Kerr media with a Gaussian response profile.

The dependence of the growth rate on the wave number and degree of nonlocality $\sigma$ is displayed in Fig. 4. Evidently, as degree of the nonlocality increases the MI bandwidth shrinks and the maximum growth rate decreases. Thus nonlocality clearly tends to suppress MI in this case, as also predicted by the weakly nonlocal model.

In Fig. 5 we demonstrate the influence of nonlocality on the dynamic development of MI. To this end we integrated
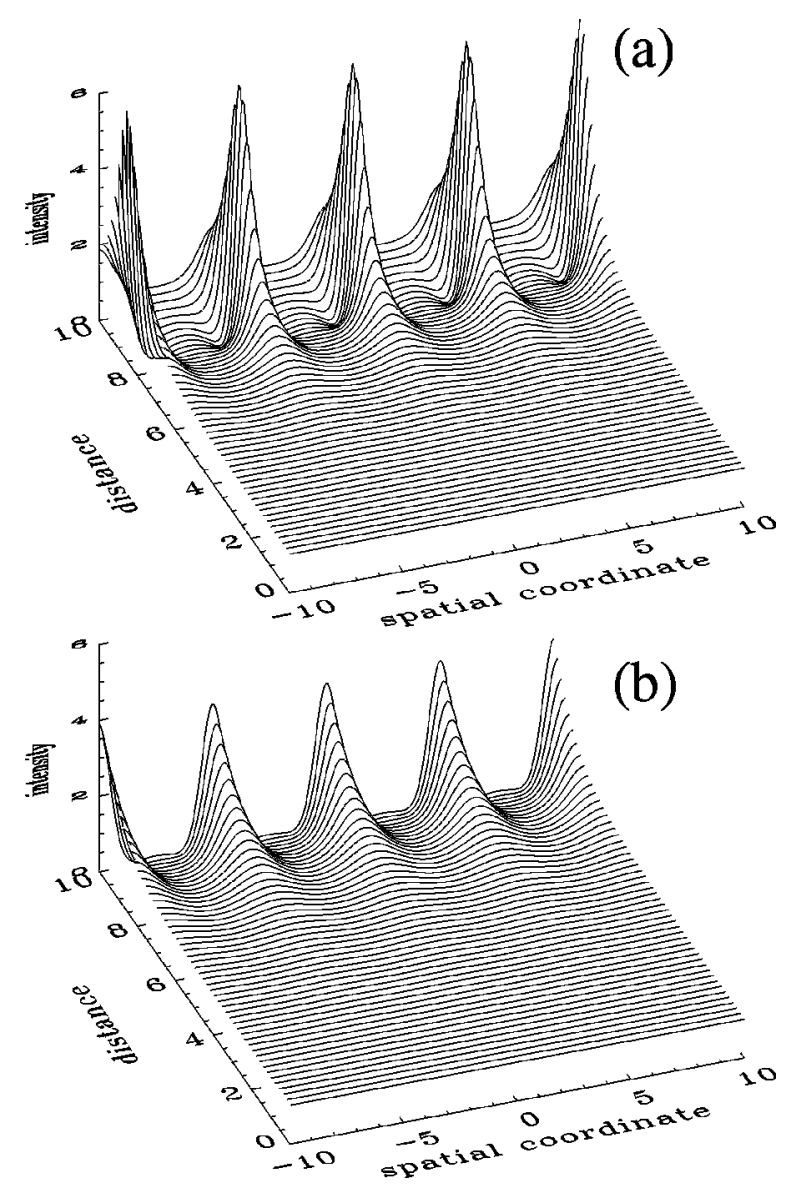

FIG. 5. Development of MI of a unit amplitude plane wave in a nonlocal self-focusing $(\mathrm{s}=1)$ medium with a Gaussian response function for (a) $\sigma=0.1$ and (b) $\sigma=1.0$. 
numerically the NLS equation (7) with the Gaussian response function (30). As before we employed the split-step Fourier method in conjunction with the finite difference for calculating the convolution integral in Eq. (7). As an initial conditions we used a unit intensity plane wave with imposed weak periodic perturbation

$$
\psi(x, z=0)=1+10^{-4} \cos (1.5 x) .
$$

It is evident that increasing the degree of nonlocality $\sigma$ suppresses the instability by lowering the gain.

\section{B. Exponential response function}

Another interesting and important example involves the exponential response function

$$
R(x)=\frac{1}{2 \sigma} \exp \left[-\frac{|x|}{\sigma}\right],
$$

for which $\gamma=2 \sigma^{2}$, with $\sigma$ again determining the degree of nonlocality. The importance of this particular response lies in the fact that it applies exactly to media with a nonlinearity [i.e., nonlinear refractive index $\Delta n(x)$ ] described by the diffusion equation

$$
-\sigma^{2} \frac{\partial^{2} \Delta n}{\partial x^{2}}+\Delta n=|\psi|^{2}
$$

The equivalence between the nonlocal nonlinearity (1) with the exponential response (35) and the diffusion model (36) is easily shown by Fourier transformation.

As discussed by Litvak et al. [18] the model (36) describes the nonlinear thermal self-focusing of electromagnetic waves in a weakly ionized plasma. In this particular case $\Delta n$ corresponds to the change of the plasma temperature. From the Fourier transform

$$
\hat{R}(k)=\frac{1}{1+\sigma^{2} k^{2}},
$$

we obtain the eigenvalue equation

$$
\lambda^{2}=-k^{2} \rho_{0}\left[\alpha k^{2}-s \frac{1}{1+(\sigma k)^{2}}\right],
$$

which coincides with that derived in Ref. [18] [Eq. (4.1) in this reference]. The dispersion relation (38) imposes MI properties similar to those found for the Gaussian response. In particular, it follows that in the self-defocusing regime $(s=-1)$ the plane wave solution is always modulationally stable. On the other hand, in the self-focusing case plane wave develops transverse instability with the growth rate given by

$$
|\operatorname{Re} \lambda|=|k| \sqrt{\rho_{0}} \sqrt{\frac{1}{1+(\sigma k)^{2}}-\alpha k^{2}} .
$$

In Fig. 6 we show the MI growth rate versus the wave num-

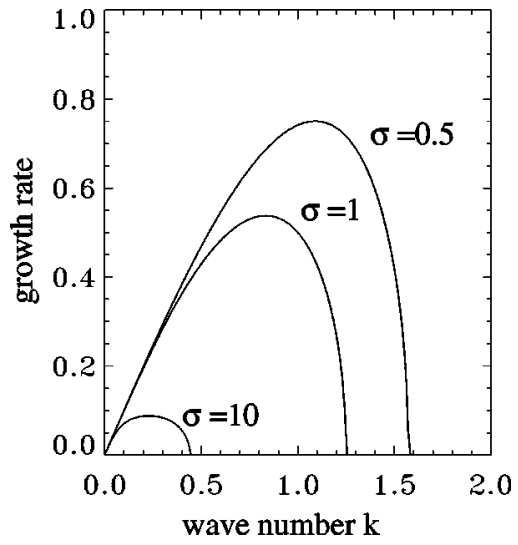

FIG. 6. MI gain profiles for $\rho_{0}=1$ in nonlocal self-focusing $(\mathrm{s}=1)$ media with an exponential response function.

ber for a few values of the nonlocality parameter $\sigma$. As for the Gaussian response the nonlocality tends to suppress MI by narrowing the gain bandwidth and decreasing the maximum gain. However, only in the extreme limit of an infinitely nonlocal response $(\sigma \rightarrow \infty)$ does MI completely disappear, as the medium no longer exhibits any nonlinearity.

\section{Rectangular response function}

Finally we consider the rectangular response function

$$
R(x)=\left\{\begin{array}{ccc}
\frac{1}{2 \sigma} & \text { for } & |x| \leqslant \sigma \\
0 & \text { for } & |x|>\sigma,
\end{array}\right.
$$

for which $\gamma=\sigma^{2} / 3$. From the Fourier transform

$$
\hat{R}(k)=\frac{\sin (k \sigma)}{k \sigma},
$$

we obtain the eigenvalue equation

$$
\lambda^{2}=-k^{2} \rho_{0}\left[\alpha k^{2}-s \frac{\sin (k \sigma)}{k \sigma}\right] .
$$

In the focusing case one again finds that there is always (for any value of $\alpha$ and $\sigma$ ) a finite bandwidth MI gain band symmetrically located about the origin in $k$ space, i.e., for $|k| \leqslant k_{\mathrm{cr}}$, where

$$
\alpha k_{\mathrm{cr}}^{2}=\frac{\sin \left(k_{\mathrm{cr}} \sigma\right)}{k_{\mathrm{cr}} \sigma},
$$

This is illustrated in Fig. 7, where we show the gain profiles for several degrees of nonlocality $\sigma$. Again MI is being suppressed by the nonlocality, but now additional MI bands appear for large values of $\sigma$ (see $\sigma=20$ ).

In the defocusing case a careful analysis reveals that for large and moderate values of the parameter $\alpha / \sigma^{2}$, i.e., small and moderate values of $\rho_{0} \sigma^{2}$, plane waves are modulationally stable, while in the opposite regime one or more MI bands appear, as shown in Fig. 8. This instability is caused 


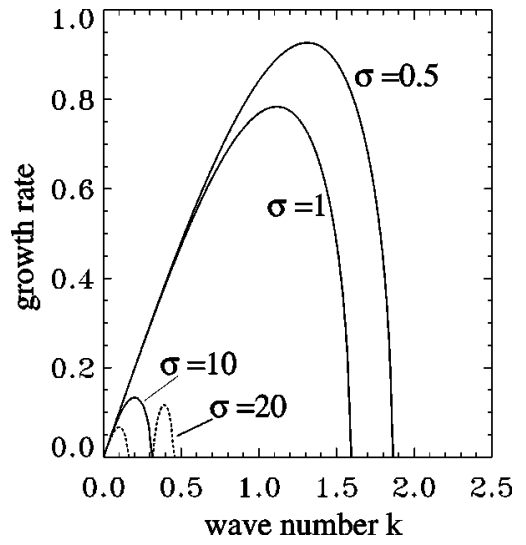

FIG. 7. MI gain profiles for $\rho_{0}=1$ in self-focusing $(\mathrm{s}=1)$ nonlocal media with a rectangular response function.

by the negative-sign bands of the $\sin (x)$-profile Fourier transform (41), with the first instability band being in the region $\pi \leqslant k \sigma \leqslant 2 \pi$. More specifically the instability appears when the background intensity exceeds the threshold value

$$
\rho_{0}^{\mathrm{th}}>21.05 / \sigma^{2}
$$

\section{CONCLUSIONS}

We studied the modulational stability properties of plane waves in nonlinear media with a general nonlocal Kerr nonlinearity, described by a nonlocal NLS equation. We derived an analytical expression for the growth rate of the instability for arbitrary response profiles, i.e., for arbitrary degrees of nonlocality. We found that in the self-focusing case the plane wave solution is always unstable-the nonlocality tends to suppress the instability, independently of the form of the response function, but can never suppress it completely.

In self-defocusing media the stability properties depend strongly on the specific form of the nonlocal response. For any strictly positive continuous response profile all plane

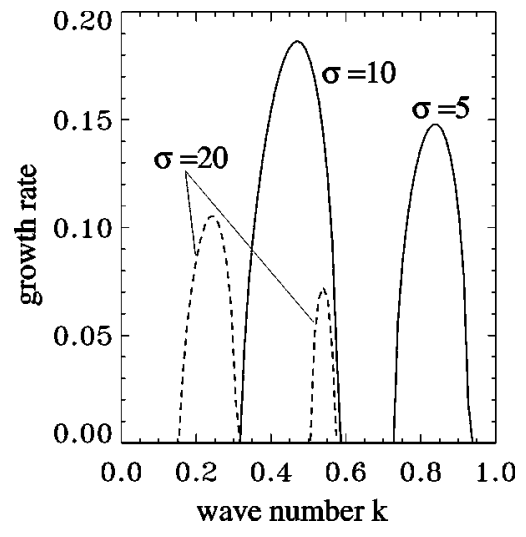

FIG. 8. MI gain profiles for $\rho_{0}=1$ in self-defocusing $(\mathrm{s}=-1)$ nonlocal media with a rectangular response function.

waves are stable for any degree of nonlocality. However, exotic profiles with jump discontinuities like the rectangular response, may actually promote MI when the intensity is sufficiently high.

In the so-called weakly nonlocal limit (narrow response function compared to the intensity profile) we find that plane waves become modulationally unstable if their intensity exceeds a certain critical value. However, we show that this surprising prediction of MI in the defocusing case can never apply to the full nonlocal model with a smooth response profile. Thus one should be cautious with applying results of the simple weakly nonlocal model to the real general case, in particular when studying MI and dark solitons.

\section{ACKNOWLEDGMENTS}

This work was supported by the Danish Technical Research Council (STVF-Talent Grant No. 5600-00-0355), the Danish Natural Sciences Foundation (SNF-Grant No. 9903273), and the Graduate School in Nonlinear Science (The Danish Research Academy).
[1] T.B. Benjamin and J.E. Feir, J. Fluid Mech. 27, 417 (1967).

[2] A. Hasegawa, Plasma Instabilities and Nonlinear Effects (Springer-Verlag, Heidelberg, 1975).

[3] L.A. Ostrovskii, Zh. Éksp. Teor. Fiz. 51, 1189 (1966) [Sov. Phys. JETP 24, 797 (1967)].

[4] V.I. Bespalov and V.I. Talanov, Pis'ma Zh. Éksp. Teor. Fiz. Rad. 3, 471 (1966) [JETP Lett. 3, 307 (1966)]; V.I. Karpman, ibid. 6, 829 (1967) [ibid. 6, 277 (1967)].

[5] Yu.S. Kivshar and M. Peyrard, Phys. Rev. A 46, 3198 (1992).

[6] P.D. Miller and O. Bang, Phys. Rev. E 57, 6038 (1998).

[7] M.J. Potasek, Opt. Lett. 12, 921 (1987).

[8] Yu.S. Kivshar, D. Anderson, and M. Lisak, Phys. Scr. 47, 679 (1993).

[9] M. Soljacic, M. Segev, T. Coskun, D.N. Christodoulides, and A. Vishwanath, Phys. Rev. Lett. 84, 467 (2000).

[10] J. Wyller, Physica D (to be published).

[11] J.P. Gordon, R.C. Leite, R.S. Moore, S.P. Porto, and J.R.
Whinnery, J. Appl. Phys. 36, 3 (1965).

[12] S. Akhmanov, D.P. Krindach, A.V. Migulin, A.P. Sukhorukov, and R.V. Khokhlov, IEEE J. Quantum Electron. QE-4, 568 (1968).

[13] M. Horowitz, R. Daisy, O. Werner, and B. Fischer, Opt. Lett. 17, 475 (1992).

[14] D. Suter and T. Blasberg, Phys. Rev. A 48, 4583 (1993).

[15] M. Porkolab and M.V. Goldman, Phys. Fluids 19, 872 (1976).

[16] A.G. Litvak and A.M. Sergeev, Pis'ma Zh. Éksp. Teor. Fiz. 27, 549 (1978) [JETP Lett. 27, 517 (1978)].

[17] T.A. Davydova and A.I. Fishchuk, Ukr. Phys. J. 40, 487 (1995).

[18] A.G. Litvak, V.A. Mironov, G.M. Fraiman, and A.D. Yunakovskii, Fiz. Plazmy 1, 60 (1975) [Sov. J. Plasma Phys. 1, 31 (1975)].

[19] H.L. Pecseli and J.J. Rasmussen, Plasma Phys. 22, 421 (1980).

[20] F. Dalfovo, S. Giorgini, L.P. Pitaevskii, and S. Stringari, Rev. 
Mod. Phys. 71, 463 (1999); K. Goral, K. Rzazewski, and T. Pfau, Phys. Rev. A 61, 051601(R) (2000); V.M. Perez-Garcia, V.V. Konotop, and J.J. Garcia-Ripoll, Phys. Rev. E 62, 4300 (2000).

[21] A. Parola, L. Salasnich, and L. Reatto, Phys. Rev. A 57, R3180 (1998).
[22] W. Krolikowski and O. Bang, Phys. Rev. E 63, 016610 (2001).

[23] X. Wang, D.W. Brown, K. Lindenberg, and B.J. West, Phys. Rev. A 37, 3557 (1988).

[24] A. Nakamura, J. Phys. Soc. Jpn. 42, 1824 (1977).

[25] A. Snyder and J. Mitchell, Science 276, 1538 (1997). 